\title{
DIFERENCIAS EN LAS CIRCUNSTANCIAS DE RIESGO AL VOLANTE EN UNIVERSITARIOS GUATEMALTECOS Y ESPAÑOLES
}

\author{
Sandra Rodríguez Guzmán ${ }^{1, a}$, Eladio Jiménez Mejías ${ }^{2, a}$, Virginia Martínez Ruiz²,a, \\ Marta Cecilia Jaramillo Mejía ${ }^{3, b}$
}

\begin{abstract}
RESUMEN
Objetivos. Comparar las diferencias en las circunstancias de riesgo al volante en universitarios de Guatemala y España. Materiales y métodos. Estudio transversal realizado durante el 2007 a 2011 en una muestra de 2130 conductores (1016 en Guatemala y 1114 en España), quienes respondieron un cuestionario autoadministrado que valoraba: patrones de movilidad, uso de dispositivos de seguridad, estilos de conducción e implicación en accidentes de tránsito. Resultados. Los estudiantes de Guatemala se implicaron con mayor frecuencia en circunstancias de riesgo como hablar por el teléfono móvil $(74,4 \%$ vs $24,3 \%)$, distraerse $(47,1 \%$ vs $18,8 \%)$ o no usar el cinturón de seguridad $(23,9 \%$ vs $5,9 \%)$ con respecto a los españoles; en el análisis ajustado el reporte de haber tenido algún accidente fue 4,8 veces mayor (IC 95\% 3,1-7,4) en universitarios de Guatemala. Conclusiones. Existen factores dependientes del vehículo, entorno físico y de índole social, que podrían tener un rol importante en las diferencias detectadas en ambas poblaciones.
\end{abstract}

Palabras clave: Accidentes de tránsito; Conducta de riesgo; Estudiantes de salud pública (fuente: DeCS BIREME)

\section{DIFFERENCES IN MOBILITY, TRAFFIC ACCIDENTS AND ASSOCIATED CIRCUMSTANCES IN GUATEMALAN AND SPANISH UNIVERSITY STUDENTS}

\begin{abstract}
Objective. The purpose of this study was to compare the frequency of involvement in risky driving circumstances between Guatemalan and Spanish university students and identify in both populations the differences between the involvement in such circumstances and road crashes. Materials and methods. A cross sectional study was conducted during the academic courses 2007 to 2011 on a sample of 2130 drivers (1 016 in Guatemala and 1114 in Spain), who completed a self-administered questionnaire that assessed: mobility patterns, use of safety devices, driving styles and involvement in road traffic crashes. Results: Furthermore, they were involved more frequently in almost all the risky-driving circumstances compared with Spanish students, principally in: mobile use (74.4\% versus $24.3 \%)$, distraction (47.1\% versus $18.8 \%)$ or not using seatbelt ( $23.9 \%$ vs 5.9). Finally, the adjusted analysis yields an accident rate 4.8 times higher among Guatemalans $(\mathrm{Cl}$ 95\% 3.1-7.4). Conclusions: Considering the factors more frequently associated with suffer road traffic crashes dependent on human factor, it is noted physical and social factors as well as that the car-dependent issues, must play an important role in the marked differences detected in both populations.
\end{abstract}

Key words: Accidents, traffic; Risk-taking; Students, public health (source MeSH)

\section{INTRODUCCIÓN}

Cada año más de 1,2 millones de personas pierden la vida y entre 20 a 50 millones resultan heridas a causa de las lesiones por accidentes de tránsito, lo que según la Organización Mundial de la Salud (OMS) sería la novena causa de mortalidad en todo el mundo. Pero lejos de ser un problema en vías de solución, su tendencia creciente las convertirá en la quinta causa de mortalidad mundial en el año $2030{ }^{(1)}$.

Es conocido que el factor humano es el principal determinante de la accidentalidad por tránsito en conductores de automóviles ${ }^{(2,3)}$, particularmente en

\footnotetext{
Universidad de San Carlos. Guatemala

Universidad de Granada. España

Universidad Icesi. Colombia

a Máster en Investigaciones y Avances en Medicina Preventiva y Salud Pública; ${ }^{\mathrm{b}}$ profesora Departamento de Gestión Organizacional. Recibido: 18-02-15 Aprobado: 23-07-15
} 
jóvenes, los que presentan factores de riesgo ligados a la inexperiencia y a la falta de percepción del riesgo (4-7). Sin embargo, es razonable asumir que el impacto de estos factores dependerá de su interacción con los determinantes de la accidentalidad: el vehículo, las infraestructuras viales y el entorno social y legal ${ }^{(8)}$. En definitiva, se plantea que la implicación en conductas de riesgo y su asociación con la accidentalidad entre jóvenes conductores cambia en función del entorno. Obviamente, la respuesta a esta pregunta, de indudable interés a la hora de orientar las políticas de seguridad vial, no es sencilla, y el presente estudio constituye tan solo una primera aproximación.

El Grupo de Investigación de la Epidemiología de las Lesiones por Tránsito del Departamento de Medicina Preventiva y Salud Pública de la Universidad de Granada (España) desarrolló, entre 2007 y 2010, el cuestionario MATCA (Movilidad, Accidentalidad por Tránsito y Circunstancias Asociadas), orientado, entre otros aspectos, a cuantificar la frecuencia de involucrarse por parte de los conductores de coche en circunstancias de conducción de riesgo, así como la asociación entre dichas circunstancias y la accidentalidad autodeclarada. Estamos ante dos poblaciones de conductores relativamente comparables con respecto a su rango de edades y a su posición en la escala socioeconómica de cada país, pero ubicados en dos entornos de conducción sustancialmente diferentes: España es un país desarrollado, con un sistema de circulación (vehículos, infraestructuras viales, políticas de seguridad vial y legislación) equiparable al de los países de su entorno. Por su parte, Guatemala es un país en desarrollo, con un sistema de circulación acorde a su contexto socioeconómico, menos desarrollado que el español (9).

Indudablemente, estas diferencias se traducen en las correspondientes tasas de mortalidad por tránsito reportadas en ambos países: 8,8/100 000 habs. en España frente a 14,7 en Guatemala, según datos de $2007^{(10,11)}$. Estas diferencias son aun mayores si tenemos en cuenta la no centralización de los datos de siniestralidad vial estatal y la tendencia a su infradeclaración en Guatemala, según el Consejo de Prevención de Accidentes y Educación Vial Guatemalteco (CONPREVE) ${ }^{(12,13)}$.

En este contexto y dada la escasez de estudios que analicen las circunstancias de riesgo con la accidentalidad por tránsito, tras la aplicación del cuestionario MATCA en las dos poblaciones de estudiantes universitarios antes mencionadas, con el objetivo de identificar la existencia de diferencias entre ambas poblaciones con respecto a la magnitud de riesgo con la accidentalidad por tránsito la asociación entre estas autodeclarada.

\section{MATERIALES Y MÉTODOS}

\section{DISEÑO Y POBLACIÒN DE ESTUDIO}

Estudio de tipo transversal, tomando como población a los alumnos de grado del Departamento de Salud Pública de la Universidad de San Carlos en Guatemala, durante el curso académico 2010-2011 y del Departamento de Medicina Preventiva y Salud Pública de la Universidad de Granada en España, durante los cursos académicos 2007-2008 a 2009-2010. Durante algún día de las dos primeras semanas lectivas, el profesor informó a los alumnos sobre el objetivo del estudio y repartió un cuestionario autoadministrado de cumplimentación voluntaria.

\section{LA MUESTRA}

Dado que una de las pretensiones futuras de este estudio es la implementación y seguimiento de una cohorte prospectiva de usuarios de la vía, para el presente trabajo no se predeterminó un tamaño de muestra mínimo, por lo que estuvo constituida por 1016 estudiantes de Guatemala y 1014 estudiantes de Granada que manifestaron haber conducido un automóvil durante el año anterior a la encuesta.

\section{EL INSTRUMENTO}

El instrumento empleado fue el Cuestionario sobre Movilidad, Accidentalidad por Tránsito y Circunstancias Asociadas (MATCA), que presenta las siguientes partes: 1). Datos demográficos; 2). Intensidad de exposición ( $\mathrm{km}$ recorridos al año, estratificados originalmente en ocho categorías y posteriormente reagrupados en tres para los conductores de coche: <1000, 1000-9999 y 10 000 o más km); 3). Datos para conductores de vehículos a motor: edad de obtención del permiso, velocidad percibida, calidad de conducción percibida e implicación durante el mes anterior a la encuesta, en 28 diferentes circunstancias al volante de respuesta dicotómica $(0=$ No y 1=Sí). A partir de esta última información se construyó un índice de riesgo, sumando la implicación en aquellas circunstancias más claramente asociadas con un mayor riesgo de sufrir un accidente, según la literatura; 4). Accidentes de tránsito sufridos en el año anterior a la encuesta $y$, para el último accidente sufrido, diversas características del mismo (tipo de usuario, severidad, responsabilidad).

De las cuatro partes del cuestionario, la fiabilidad del segundo fue previamente medida en una muestra de 90 alumnos y médicos residentes de primer año del Hospital Clínico Universitario de Granada (España), luego se contrastó con la obtenida a través de una versión modificada del Driving Habits Questionnaire, desarrollado 
por Owsley, Stalvey, Wells y Sloane (1999) ${ }^{(14)}$, de ello se obtuvo una concordancia aceptable entre ambos instrumentos (coeficiente kappa=0,846). Con respecto a la parte 3 , la validez de convergencia y de criterio de las 28 circunstancias planteadas (seleccionadas por un panel de expertos a partir de una revisión exhaustiva de la bibliografía) ${ }^{(15)}$, ha sido validada previamente a partir de la muestra de estudiantes universitarios de Granada (16). Posteriormente, dicho cuestionario, con ligeras modificaciones, ha sido aplicado a una muestra de alumnos de la Universidad de San Carlos, en Ciudad de Guatemala.

\section{LAS VARIABLES}

Se consideraron alternativamente tres grupos de variables dependientes: intensidad de exposición como conductor de coche, implicación en circunstancias de conducción de riesgo y accidentalidad. Con respecto a cada grupo de variables, el análisis se ha desglosado en describir las diferencias en la distribución de cada grupo de variables en función del país de origen y en analizar la influencia del país en cada uno de los siguientes elementos: intensidad de exposición, implicación en circunstancias de conducción de riesgo y haber sufrido un accidente. A tal efecto se han construido modelos de regresión en los que, alternativamente, se han ido considerando las variables anteriores como variables dependientes del modelo y, como variable de exposición, el país.

\section{ANÁLISIS DE LOS DATOS}

Se construyeron modelos ajustados añadiendo al país, el sexo, la edad, la antigüedad del permiso de conducir y las variables correspondientes a la exposición y la implicación en circunstancias de conducción. Dependiendo del tipo de variable dependiente se aplicaron modelos de regresión logística (para la frecuencia de haber sufrido algún accidente de tránsito y para la implicación en cada una de las circunstancias de conducción), de regresión politómica nominal (para los tres niveles de intensidad de exposición y los tres niveles de velocidad y calidad percibidas), y de regresión lineal (para el número de circunstancias de conducción de riesgo referidas).

Finalmente, para valorar si la asociación entre la exposición y las circunstancias de riesgo se asociaban con una magnitud diferente en función del país considerado, se construyeron sendos modelos de regresión logística (uno por país), en los que la variable dependiente fue la accidentalidad $\mathrm{y}$, como variables independientes, se incluyeron la intensidad de exposición, la velocidad y la calidad percibidas, el número de circunstancias de riesgo referidas por cada conductor, junto con el sexo, la edad y la antigüedad en el permiso de conducir. El software empleado para todos los análisis fue el paquete estadístico Stata $11.0^{(17)}$.

\section{CONSIDERACIONES ÉTICAS}

El estudio contó con la aprobación de los comités de ética de las dos universidades implicadas para la utilización de datos personales de los estudiantes. Además, a los participantes se les solicitó verbalmente y por escrito el consentimiento para disponer de sus datos personales (al menos un correo electrónico de contacto). Únicamente la Universidad de Granada que lidera el presente estudio, tiene acceso a los datos personales de los participantes que serán empleados en los próximos años para el seguimiento de la cohorte.

\section{RESULTADOS}

La proporción de varones fue mayor entre los estudiantes guatemaltecos que entre los españoles (46,9\% vs $26,9 \%$ ). Los conductores guatemaltecos fueron en general más jóvenes con respecto a los españoles (media de edad 20,7 años vs 23,3, respectivamente). La media de años en posesión del permiso de coche fue también menor entre los estudiantes de Guatemala (3,3 vs 4,2 de los españoles). En lo referente a la intensidad de exposición, se observó que los conductores guatemaltecos refirieron un mayor número de kilómetros recorridos durante el último año, a diferencia de los españoles $(p<0,001)$ (Tabla 1).

Tabla 1. Características de los universitarios según nacionalidad

\begin{tabular}{|c|c|c|c|c|c|}
\hline \multirow{2}{*}{ Variables } & \multicolumn{2}{|c|}{ Guatemala } & \multicolumn{2}{|c|}{ España } & \multirow{2}{*}{ Valor $p$} \\
\hline & $\mathbf{n}$ & $(\%)$ & $\mathbf{n}$ & $(\%)$ & \\
\hline \multicolumn{6}{|l|}{ Sexo } \\
\hline Varón & 474 & $(46,9)$ & 296 & $(26,9)$ & $<0,001$ \\
\hline Mujer & 537 & $(53,1)$ & 806 & $(73,1)$ & \\
\hline \multicolumn{6}{|l|}{ Edad (años) } \\
\hline$<20$ & 274 & $(28,3)$ & 71 & $(6,9)$ & $<0,001$ \\
\hline $20-22$ & 559 & $(57,8)$ & 440 & $(42,5)$ & \\
\hline $23-24$ & 95 & $(9,8)$ & 323 & $(31,2)$ & \\
\hline$\geq 25$ & 40 & $(4,1)$ & 202 & $(19,5)$ & \\
\hline \multicolumn{6}{|c|}{ Antigüedad en el permiso de conducir (años) } \\
\hline$<1$ & 173 & $(19,4)$ & 149 & $(15,1)$ & 0,013 \\
\hline 2 & 181 & $(20,3)$ & 180 & $(18,3)$ & \\
\hline 3 & 192 & $(21,5)$ & 201 & $(20,4)$ & \\
\hline 4 & 136 & $(15,2)$ & 165 & $(16,7)$ & \\
\hline$\geq 5$ & 212 & $(23,7)$ & 291 & $(29,5)$ & \\
\hline \multicolumn{6}{|c|}{ Intensidad de exposición (km/año) } \\
\hline$<1000$ & 145 & $(14,3)$ & 558 & $(50,1)$ & $<0,001$ \\
\hline $1000-9999$ & 348 & $(34,2)$ & 371 & $(33,3)$ & \\
\hline$\geq 10000$ & 523 & $(51,5)$ & 185 & $(16,6)$ & \\
\hline
\end{tabular}




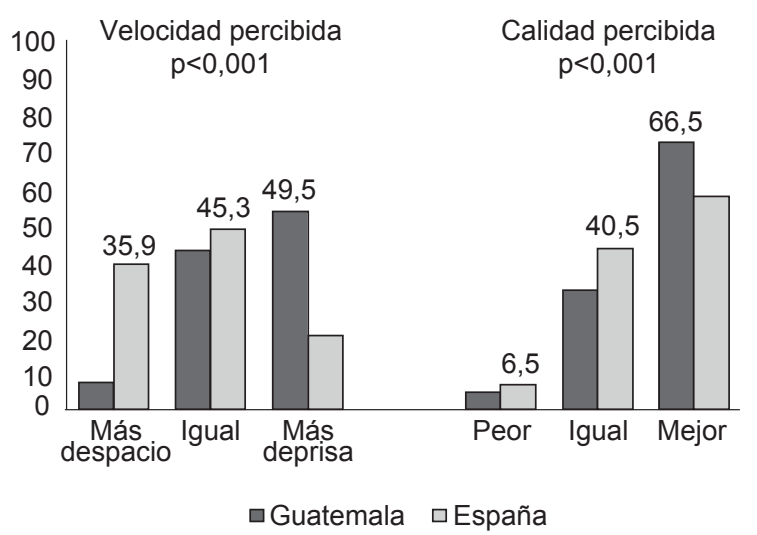

Figura 1. Velocidad y calidad percibidas por los conductores según nacionalidad

La Figura 1 muestra las distribuciones de la velocidad y la calidad percibidas por los conductores según su nacionalidad. Se observa que los conductores guatemaltecos fueron los que con mayor frecuencia manifestaron conducir más deprisa y se percibieron como mejores al volante, con respecto a los españoles. Además, la frecuencia de implicación en casi todas las circunstancias de conducción propuestas fue sensiblemente mayor en los guatemaltecos (Tabla 2).
Las mayores diferencias se dieron, a favor de éstos últimos, en las siguientes circunstancias: conducir hablando por teléfono móvil, distraerse al volante, comer mientras se conduce, adelantar por la derecha estando prohibido y no usar el cinturón de seguridad. Lo contrario ocurrió para las circunstancias conducir en autopista y no respetar un paso de peatones, ambas más frecuentes entre los españoles.

Un $24,7 \%$ de los guatemaltecos manifestaron haber sufrido uno o más accidentes en el último año viajando como conductores de coche, frente a un $4,9 \%$ de los españoles. La OR cruda para dicha asociación fue de 6,4 (IC 95\%: 4,7-8,8) en guatemaltecos con respecto a los españoles.

En la Tabla 3 se muestran los resultados de los análisis multivariable para cuantificar el efecto ajustado de la nacionalidad sobre los restantes grupos de variables. En ella puede comprobarse como, aún tras ajustar por sexo, edad y antigüedad del permiso, la nacionalidad guatemalteca se asoció con una mayor intensidad de exposición. Tras ajustar por exposición, ser guatemalteco se asoció con una mayor velocidad y calidad percibidas al volante, así con la implicación en un mayor número de circunstancias de riesgo al volante. Finalmente, el análisis ajustado por todas las variables consideradas,

Tabla 2. Circunstancias de riesgo al volante durante el último mes según nacionalidad

\begin{tabular}{|c|c|c|c|c|c|}
\hline \multirow{2}{*}{ Circunstancias } & \multicolumn{2}{|c|}{ Guatemala } & \multicolumn{2}{|c|}{ España } & \multirow{2}{*}{$\begin{array}{c}\text { Valor } \\
p\end{array}$} \\
\hline & $\mathrm{N}=1016$ & $(\%)$ & $n=1114$ & $(\%)$ & \\
\hline Conducir de noche & 838 & $(83,1)$ & 885 & $(79,4)$ & 0,030 \\
\hline Conducir por encima de la velocidad permitida & 531 & $(52,6)$ & 521 & $(46,7)$ & 0,007 \\
\hline Conducir con sueño & 534 & $(52,9)$ & 239 & $(21,4)$ & $<0,001$ \\
\hline No respetar un semáforo & 228 & $(22,6)$ & 196 & $(17,6)$ & 0,004 \\
\hline Conducir después de haber consumido alcohol & 320 & $(31,7)$ & 161 & $(14,5)$ & $<0,001$ \\
\hline Conducir solo & 920 & $(91,5)$ & 875 & $(78,5)$ & $<0,001$ \\
\hline Conducir después de haber consumido drogas & 23 & $(2,3)$ & 25 & $(2,2)$ & 0,954 \\
\hline Conducir bajo clima adverso & 883 & $(87,8)$ & 801 & $(71,9)$ & $<0,001$ \\
\hline Conducir y hablar por el móvil a la vez & 747 & $(74,4)$ & 271 & $(24,3)$ & $<0,001$ \\
\hline No respetar una señal de STOP & 176 & $(17,5)$ & 142 & $(12,7)$ & 0,002 \\
\hline Conducir sin cinturón & 241 & $(23,9)$ & 66 & $(5,9)$ & $<0,001$ \\
\hline Conducir en autopista & 485 & $(48,3)$ & 873 & $(78,3)$ & $<0,001$ \\
\hline La policía me ha puesto una multa & 191 & $(19,0)$ & 39 & $(3,5)$ & $<0,001$ \\
\hline Tener un accidente sin lesionados & 217 & $(21,5)$ & 37 & $(3,3)$ & $<0,001$ \\
\hline Tener un accidente con lesionados & 29 & $(2,9)$ & 2 & $(0,2)$ & $<0,001$ \\
\hline Conducir con síntomas de embriaguez & 168 & $(16,7)$ & 27 & $(2,4)$ & $<0,001$ \\
\hline No respetar un paso de peatones & 155 & $(15,4)$ & 379 & $(34,0)$ & $<0,001$ \\
\hline Un acompañante me ha dicho que corro mucho & 309 & $(30,7)$ & 165 & $(14,8)$ & $<0,001$ \\
\hline Me he distraído al volante & 474 & $(47,1)$ & 210 & $(18,8)$ & $<0,001$ \\
\hline Fumar mientras conduzco & 212 & $(21,1)$ & 91 & $(8,2)$ & $<0,001$ \\
\hline Escuchar la radio y cambiar de emisora & 832 & $(82,5)$ & 804 & $(72,2)$ & $<0,001$ \\
\hline Cambiar el CD mientras conduzco & 510 & $(50,7)$ & 410 & $(36,8)$ & $<0,001$ \\
\hline Comer mientras conduzco & 614 & $(60,9)$ & 166 & $(14,9)$ & $<0,001$ \\
\hline Conducir más de dos horas sin descansar & 385 & $(38,3)$ & 229 & $(20,6)$ & $<0,001$ \\
\hline Pitar al de delante en un ceda el paso o semáforo & 245 & $(24,3)$ & 129 & $(11,6)$ & $<0,001$ \\
\hline Discutir con otros conductores & 251 & $(25,0)$ & 145 & $(13,0)$ & $<0,001$ \\
\hline Adelantar por la derecha estando prohibido & 255 & $(25,3)$ & 74 & $(6,6)$ & $<0,001$ \\
\hline
\end{tabular}


Tabla 3. Asociación de la nacionalidad guatemalteca con la intensidad de exposición, la velocidad y calidad percibidas, el no uso de cinturón, el número de circunstancias de conducción y haber sufrido algún accidente

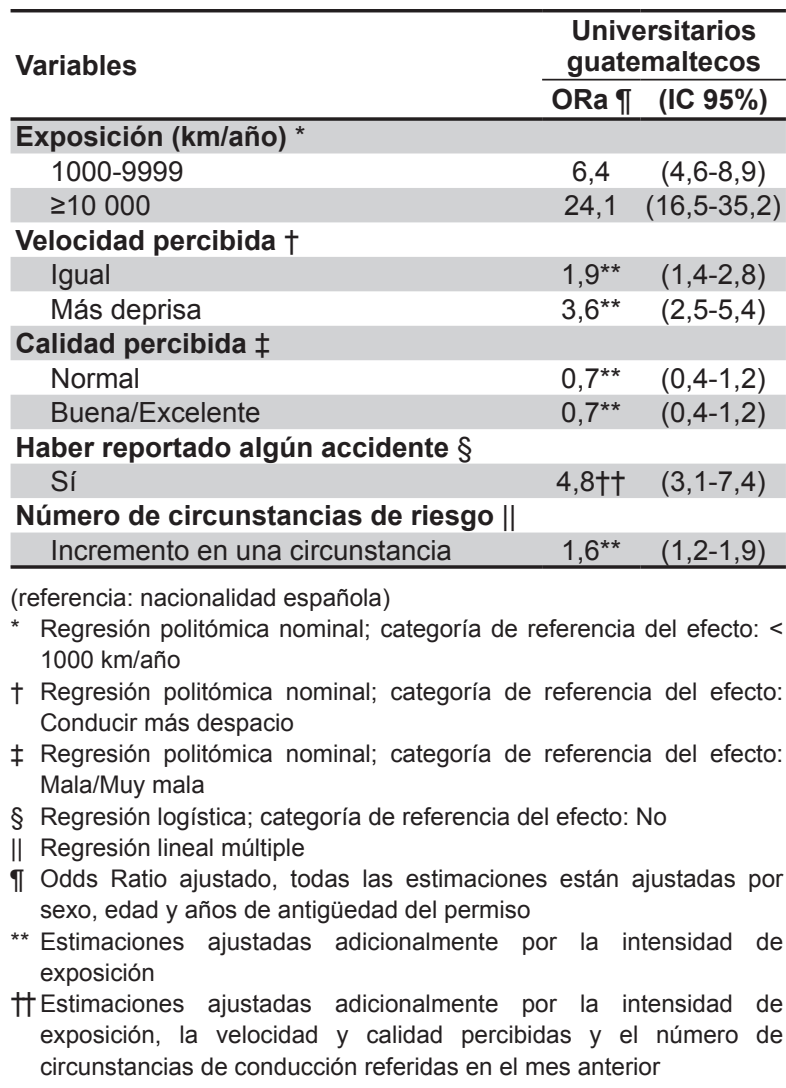

arrojó una frecuencia de reporte de accidentes 4,8 veces mayor en los guatemaltecos con respecto a los españoles.

En la Tabla 4 se presentan los dos modelos para valorar el efecto de la exposición, la velocidad y calidad percibidas, junto con el número de circunstancias de

Tabla 4. Regresión logística: asociación entre número de circunstancias de riesgo acumuladas en el último mes percibidas por universitarios de España y Guatemala

\begin{tabular}{|c|c|c|c|c|}
\hline \multirow{2}{*}{ Variables } & \multicolumn{2}{|c|}{ España } & \multicolumn{2}{|c|}{ Guatemala } \\
\hline & ORa* & (IC 95\%) & ORa* & (IC 95\%) \\
\hline \multicolumn{5}{|l|}{ Exposición (km/año) } \\
\hline $1000-9999$ & 2,7 & $(1,2-6,3)$ & 1,9 & $(0,9-3,6)$ \\
\hline$\geq 10000$ & 3,1 & $(1,1-8,7)$ & 1,5 & $(0,8-2,8)$ \\
\hline \multicolumn{5}{|l|}{ Velocidad percibida } \\
\hline Igual & 2,4 & $(0,9-6,3)$ & 0,9 & $(0,5-1,7)$ \\
\hline Más deprisa & 2,6 & $(0,9-8,0)$ & 0,8 & $(0,4-1,4)$ \\
\hline \multicolumn{5}{|l|}{ Calidad percibida } \\
\hline Normal & 0,4 & $(0,1-1,7)$ & 0,9 & $(0,4-2,4)$ \\
\hline Buena/Excelente & 0,5 & $(0,1-1,9)$ & 0,6 & $(0,3-1,6)$ \\
\hline \multicolumn{5}{|c|}{ Número de circunstancias de riesgo } \\
\hline Cada circunstancia & 1,2 & $(1,0-1,3)$ & 1,1 & $(1,1-1,2)$ \\
\hline
\end{tabular}

riesgo, sobre la accidentalidad, separadamente en España y Guatemala. Puede comprobarse que existen diferencias en la magnitud de las asociaciones obtenidas en función del país. No obstante, en ambos países se observa una asociación consistente y de magnitud similar entre la accidentalidad y el número de circunstancias de riesgo, con odds ratio ajustadas de 1,2 (IC 95\%: 1,1-1,3) en España y de 1,1 (IC 95\%: 1,1-1,2) en Guatemala.

\section{DISCUSIÓN}

En España, la mayoría de los conductores presentaron bajas intensidades de exposición, hecho que se corresponde con los resultados de otros estudios en poblaciones jóvenes (18). En Guatemala, por el contrario, la mayoría de conductores presentaron intensidades de exposición elevadas (ORa=24,1 para exposiciones mayores a $10000 \mathrm{~km}$, tomando como referencia a los conductores españoles). Este hallazgo puede ser parcialmente explicado por las siguientes circunstancias que concurren en un país como Guatemala: la posibilidad de conducir con un permiso temporal de aprendizaje (19), el mayor poder adquisitivo de los estudiantes guatemaltecos (grupo minoritario y privilegiado en su país) y un transporte público muy deficitario, cuyo uso se da mayoritariamente entre los estratos socioeconómicos más desfavorecidos ${ }^{(20)}$. Todo ello explica que los colectivos con un nivel socioeconómico elevado, como el representado en nuestra muestra de universitarios guatemaltecos, tiendan a desplazarse mayoritariamente en vehículos privados.

En nuestro estudio destaca la elevada frecuencia de no uso de este dispositivo entre los conductores guatemaltecos, con respecto a los españoles. Ello puede deberse al hecho de que en Guatemala, según el Informe Panamericano de la OMS de 2009, las políticas de seguridad vial son más laxas y tienen menor aplicabilidad que en España, donde se implantaron a comienzos de los noventa y cuentan con un mayor grado de cumplimiento ${ }^{(11,21)}$.

Los conductores guatemaltecos manifestaron conducir más deprisa y percibirse como buenos o excelentes al volante, además, la frecuencia de implicarse en "circunstancias de conducción" fue considerablemente mayor que España. En particular, las diferencias fueron especialmente marcadas para conducir y hablar por el móvil, distraerse al volante, no usar el cinturón de seguridad, haber recibido una multa por parte de la policía y conducir con síntomas de embriaguez (todas ellas entre un 15 a $45 \%$ más frecuentes en guatemaltecos que en españoles). No ha sido posible encontrar trabajos que apoyen o desmientan la mayor frecuencia entre los conductores guatemaltecos de 
incurrir en situaciones de riesgo con respecto a otros jóvenes conductores. Es posible que esto se deba en parte a una mayor exposición, a unas leyes menos estrictas en materia de prevención vial y con una baja aplicabilidad, así como a una política de educación vial menos extendida ${ }^{(9)}$.

Los análisis ajustados confirmaron todas las asociaciones crudas descritas en los párrafos anteriores. Así, la nacionalidad guatemalteca se asoció con una mayor exposición, a un menor uso de dispositivos de seguridad y a una mayor implicación en circunstancias de riesgo. Finalmente, el análisis ajustado mostró que la nacionalidad guatemalteca se asoció de manera consistente con una mayor accidentalidad, por lo que puede asumirse que una parte sustancial del exceso de accidentalidad reportada entre los estudiantes de Guatemala es atribuible a los factores valorados en nuestro cuestionario. Indudablemente, los factores no recogidos en el mismo, relacionados con el vehículo y el entorno físico, social y legal, deben estar debajo de gran parte de la diferencia ajustada en la accidentalidad hallada en nuestro estudio. La implicación en circunstancias de riesgo fue la variable más claramente asociada con una mayor accidentalidad en ambos países. Este hecho, junto con elevadas intensidades de exposición, han sido los dos principales factores asociados a un mayor riesgo de sufrir un accidente de tránsito en diversos estudios ${ }^{(22-27)}$

Entre las limitaciones del estudio se encuentra, en primer lugar, su carácter transversal, que impide identificar asociaciones causales entre las variables consideradas. A dicha limitación se une el posible sesgo de selección de nuestra muestra. Las poblaciones de estudio no se seleccionaron con el objeto de que fueran comparables entre sí, sino atendiendo a otras razones, fundamentalmente basadas en la factibilidad. La posible falta de representatividad vendría determinada por los dos criterios de selección establecidos: acudir a clase y aceptar cumplimentar el cuestionario. Es complejo predecir el sentido del sesgo que pueden introducir ambos factores. Esta evidente falta de comparabilidad hace que las conclusiones que pueden establecerse a partir de nuestros resultados deben limitarse a constatar (y en su caso, cuantificar), pero no a explicar, la existencia de diferencias en los patrones de los factores potencialmente ligados a la accidentalidad por tránsito, entre estas dos poblaciones. No obstante, dado el carácter voluntario y no anónimo del cuestionario, es razonable pensar que los estudiantes tiendan a suavizar las respuestas relacionadas con la implicación en estilos de riesgo al volante lo que supondría una infraestimación de las asociaciones detectadas. Dicho esto, también es necesario tener en cuenta que, al menos, los modelos multivariantes empleados han permitido ajustar las asociaciones por los grandes determinantes de la accidentalidad por tránsito dependiente de los factores individuales: la edad, el sexo, la experiencia, la intensidad de exposición y los patrones de conducción de riesgo.

Por otro lado, el cuestionario MATCA ha sido validado en la población de estudiantes universitarios españoles encuestados, pero no así en Guatemala. En este sentido, la adaptación transcultural del cuestionario a una población teóricamente muy distinta de aquella para la que fue originalmente diseñado es más que cuestionable, este aspecto debe ser considerado en futuros estudios. Algunos indicios de esta necesidad son: respecto a la exposición, la distinta distribución de la misma en las dos poblaciones comparadas, lo que exigiría una redefinición de los puntos de corte establecidos. Además, también se hace necesario adaptar las circunstancias de conducción consideradas, pues algunas de ellas son irrelevantes en un país como Guatemala (por ejemplo, conducir por autopista o autovía). Finalmente, no es posible generalizar los resultados obtenidos a otras poblaciones distintas a las originalmente comparadas. Como ejemplo, en el caso de los estudiantes universitarios españoles (y a diferencia de lo que ocurre en Guatemala), existe un claro predominio del sexo femenino entre los estudiantes de ciencias de la salud (28).

De todo lo anteriormente expuesto se deduce que, pese a las limitaciones mencionadas, nuestros resultados, en su mayoría concordantes con lo descrito por la literatura, constituyen un buen punto de partida para ahondar en el conocimiento de las diferencias en los factores de riesgo individuales en poblaciones de conductores jóvenes procedentes de países con diferentes entornos de conducción. Su estudio podría esclarecer cuáles son las dianas más importantes sobre las que dirigir la prevención de este problema de salud pública de manera más eficiente.

Contribuciones de autoría: SRG y EJM participaron en la ideación del estudio y en los análisis, así como en la escritura del primer borrador del artículo. VMR y MCJM participaron en la consulta de fuentes bibliográficas y realizaron contribuciones al mismo. Todos los autores aprobaron la versión final del artículo tras su revisión crítica.

\section{Fuentes de financiamiento: ninguna.}

Conflictos de interés: los autores declaran que no existe conflictos de intereses. 


\section{REFERENCIAS BIBLIOGRÁFICAS}

1. Peden M, Scurfield R, Sleet D, Mohan D, Hyder A, Jarawan E, et al. World report on road traffic injury prevention. Geneva: World Health Organization; 2004.

2. Elvik R, Vaa T. Factors contributing to road accidents. En: Elvik R, Hoye A, Vaa T, Sorensen M. The Handbook of Road Safety Measures. Oxford: Emerald Group Publishing Limited; 2004. p. 29-79.

3. Petridou E, Moustaki M. Human factors in the causation of road traffic crashes. Eur J Epidemiol. 2000;16(9):819-26.

4. Shope JT, Bingham CR. Teen driving: motor-vehicle crashes and factors that contribute. Am J Prev Med. 2008 Sep;35(3 Suppl):S261-71. doi: 10.1016/j.amepre.2008.06.022.

5. Tomas Dols S, Alvarez González FJ, Llorens Aleixandre N, Vidal-Infer A, Torrijo Rodrigo MJ, ValderramaZurián JC. Predictors of driving after alcohol and drug use among adolescents in Valencia (Spain). Accid Anal Prev. 2010 Nov;42(6):2024-9. doi: 10.1016/j.aap.2010.06.013.

6. Vallaso S, Smart D, Sanson A, Harrison W, Harris A, Cockfield S, et al. Risky driving among young drivers: trends, precursors and correlates. Acc Anal Prev. 2007 May;39(3):444-58.

7. Mayorga Mogollón LE. Mortalidad por accidente de tránsito en la región vial Tunja-San Gil, Colombia, 2001. Rev Salud Publica. 2003;5(2):158-71.

8. Runyan CW. Using the Haddon matrix: introducing the third dimension. Inj Prev. 1998 Dec;4(4):302-7.

9. Montenegro Alvizurez GA. Causas de la inexistencia de una política preventiva del estado, en relación a los hechos de tránsito, originados por el consumo de alcohol y estupefacientes. Tesis para obtener el grado de Licenciatura. Facultad de Ciencias Jurídicas y Sociales, Universidad de San Carlos. Ciudad de Guatemala, Guatemala. 2008.

10. Ministerio de Sanidad, Servicios Sociales e Igualdad [Internet]. Madrid: Indicadores de Salud. [citado el 21 de abril del 2014]. Disponible en: http:// www.msssi.gob.es/estadEstudios/ estadisticas/inforRecopilaciones/ indicadoresSalud2009.htm

11. Organización Mundial de la Salud. Informe sobre el estado de la seguridad vial en la región de las Américas. Washington D.C; OPS: 2009.
12. Escobedo Qujivix JM, Sabetian Layazali PW. Perfil epidemiológico de los accidentes de tránsito urbano. Tesis para obtener el grado de Licenciatura. Facultad de Ciencias Médicas, Universidad de San Carlos de Guatemala. Ciudad de Guatemala, Guatemala. 2011.

13. Gobierno de la República de Guatemala [Internet]. Operativo Anual POA 2012. Guatemala, C.A.: Ministerio de Comunicaciones, Infraestructura y Vivienda [citado el 13 de febrero de 2014]. Disponible en: http://www. insivumeh.gob.gt /LAI\%20PDF/ Articulo\%2010/inciso\%205/PLAN OPERATIVO_ANUAL.pdf

14. Owsley C, Stalvey B, Wells J, Sloane ME. Older drivers and cataract: driving habits and crash risk. J Gerontol A Biol Sci Med Sci. 1999 Apr;54(4):M203-11.

15. Jiménez-Mejías E, Lardelli-Claret P, Amezcua-Prieto C, Jiménez-Moleón JJ. Cuestionarios sobre factores de riesgo de la exposición y la accidentalidad por tránsito en conductores. Una revisión. An Sist Sanit Navar. 2011;34(3):44352.

16. Jiménez Mejías E, Luna del Castillo JD, Amezcua Prieto C, Olvera Porcel MC, Lardelli Claret P, Jiménez Moleón JJ. Diseño y validación de un cuestionario sobre patrones de conducción de riesgo en jóvenes. Rev Esp Salud Pública. 2012;86(1):71-84.

17. StataCorp. Stata Statistical Software: Release 11.0. College Station, TX: Stata Corporation, 2009.

18. Cestac J, Paran F, Delhomme P. Young drivers' sensation seeking, subjective norms, and perceived behavioral control and their roles in predicting intention: How risk-taking motivations evolve gender and driving experience. Saf Sci. 2011;49(3):424-32.

19. Organismo ejecutivo. Ministerio de Gobernación. Reglamento de tránsito. Acuerdo gubernativo número 27398 [Internet]. Guatemala, 1998 [citado el 10 de diciembre de 2013]. Disponible en: http://www.ati.com. gt/reglamento-transito.pdf.

20. Morán Mérida A, Herrero López A, Urbina Aragón R, Bethancourt Castañeda R. El transporte colectivo urbano en el área metropolitana: hacia una solución integral. Informe final. Ciudad de Guatemala: Universidad de San Carlos de Guatemala; 2001.
21. Ministerio de Justicia e Interior. Real Decreto 13/1992, de 17 de enero, por el que se aprueba el Reglamento General de Circulación, para la aplicación y desarrollo del texto articulado de la Ley sobre Tránsito, Circulación de Vehículos a Motor y Seguridad Vial. Madrid.

22. Benjamin AL. The use of seatbelts in Port Moresby 12 years after the seatbelt legislation in Papua New Guinea. PNG Med J. 2007 Sep-Dec;50(3-4):152-6.

23. Zambon F, Fedeli U, Marchesan M, Schievano E, Ferro A, Spolaore P. Seat belt use among rear passengers: validity of self-reported versus observational measures. BMC Public Health. 2008 Jul 9;8:233. doi: 10.1186/1471-24588-233.

24. Reglamento General de Circulación, para la aplicación y desarrollo del texto articulado de la Ley sobre Tránsito, Circulación de Vehículos a Motor y Seguridad Vial. Real Decreto 13/1992 [17 de enero de 1992].

25. Nabi H, Rachid Salmi L, Lafont $S$, Chiron M, Zins M, Lagarde E. Attitudes associated with behavioral predictors of serious road traffic crashes: result from the GAZEL cohort. Inj Prev. 2007 Feb;13(1):26-31.

26. Bhuiyan MA, Spainhour LK. Contributing factors for young at fault drivers in fatal traffic crashes in Florida. Journal of Transportation Safety \& Security. 2009;1(2):152-68.

27. Iversen H, Rundmo T. Personality, risky driving and accident involvement among Norweian drivers. Pers Indiv Differ. 2002;33(8):1251-63.

28. Instituto Nacional de Estadística. Estadística de la enseñanza universitaria en España. Alumnado matriculado en estudios de primer y segundo ciclo por universidad, sexo, ciclos y ramas. Curso 2010-2011 [Internet]. [citado el 8 de febrero de 2014]. Disponible en: http://www.ine.es/

Correspondencia: Eladio Jiménez Mejías Dirección: Departamento de Medicina Preventiva y Salud Pública. Universidad de Granada. Avda. de Madrid 11, 18012, Granada, España

Teléfono: + 34 958-241000 ext.49895, Fax. $+34958-246118$

Correoelectrónico: eladiojimenez@ugr.es 